\title{
Patients with Chronic Liver Disease Suggestive of Nonalcoholic Fatty Liver Disease may be at Higher Risk for Drug Induced Liver Injury
}

\author{
Craig Lammert, MD ${ }^{1}$; Timothy Imler, MD ${ }^{1,2}$; Evgenia Teal, $\mathrm{MS}^{2}$; Naga Chalasani, MD ${ }^{1}$ \\ ${ }^{1}$ Department of Medicine, Indiana University School of Medicine, Indianapolis, IN \\ ${ }^{2}$ The Regenstrief Institute, Indianapolis, IN
}

Word count: 814 (without tables or references)

Tables: 1

Key words: DILI; DILIN; Hepatotoxicity; NAFLD

Conflict of Interest Statement: No relevant conflicts of interest to declare. Dr. Chalasani has paid consulting agreements with and research grants from several pharmaceutical companies, but they are not directly or significantly related to this epidemiological study.

Funding: There was no external funding for this study.

\author{
Corresponding Author: \\ Naga Chalasani \\ Indiana University School of Medicine \\ 702 Rotary Circle, Suite 225 \\ Indianapolis, IN 46202 \\ Phone: 317-274-6492
}

Email: nchalasa@iu.eduAbbreviations:DILI: Drug Induced Liver Injury; DILIN: Drug Induced Liver Injury Network; ICD: International Classification of Diseases; ALT: Alanine aminotransferase; CLD: Chronic Liver Disease; NAFLD: Nonalcoholic Fatty Liver Disease

Authors Emails: Craig Lammert: clammert@iu.edu; Timothy Imler: timler@iu.edu;

Evgenia Teal: eteal@regenstrief.org; Naga Chalasani: nchalasa@iu.edu Author's contributions

CL: Study concept, design, data interpretation, and manuscript preparation; TI: Study concept, design, data interpretation, and manuscript preparation; ET: Study design, data extraction, data interpretation, manuscript preparation; NC: Study concept, design, data interpretation, and manuscript preparation

This is the author's manuscript of the article published in final edited form as:

Lammert, C., Imler, T., Teal, E., \& Chalasani, N. (2018). Patients with Chronic Liver Disease Suggestive of Nonalcoholic Fatty Liver Disease may be at Higher Risk for Drug Induced Liver Injury. Clinical Gastroenterology and Hepatology. https://doi.org/10.1016/j.cgh.2018.12.013 
Lammert and colleagues (2)

\section{Introduction}

To date, there have been no studies which systematically examined if individuals with nonalcoholic fatty liver disease (NAFLD have an increased risk of drug induced liver injury. We conducted a study to test if the frequency of suspected DILI from prescription agents is higher in individuals with CLD suggestive of NAFLD.

\section{Methods}

The data for this IRB approved study were collected using the Indiana University Health Enterprise Data Warehouse. We identified three cohorts of individuals seen between January 1 , 2007 and December 31, 2017. CLD cohort suggestive of NAFLD (Suspected NAFLD cohort) had serum ALT > 45 U/L in ambulatory settings on at least two occasions occurring 624 months apart in the absence of positive anti-HCV antibody, hepatitis B surface antigen, heavy alcohol consumption, hypotension, or DILI qualifying labs. Control group 1 had ALT $\leq$ $45 \mathrm{U} / \mathrm{L}$ on at least two occasions occurring 6-24 months apart whereas Control group 2 had $\mathrm{ALT} \leq 31 \mathrm{U} / \mathrm{L}$ in men and $\mathrm{ALT} \leq 19 \mathrm{U} / \mathrm{L}$ in women on at least two occasions occurring 6-24 months apart. The three cohorts were mutually exclusive.

The three cohorts were further stratified according to exposure to one of ten select medications most frequently implicated to cause DILI in the United States. (1) Exposed patients were prescribed one of these medications within one year of meeting eligibility for inclusion in the study cohorts described above. Suspected DILI was defined based on previously published criteria $(1,2)$ with modifications: serum ALT > $200 \mathrm{U} / \mathrm{L}$ and/or serum alkaline phosphatase (Alk $\mathrm{P})>250 \mathrm{U} / \mathrm{L}$ and/or total bilirubin ( $\mathrm{T}$ Bili) $>2.5 \mathrm{mg} / \mathrm{dl}$ on at least two consecutive occasions within three months after receiving one of ten candidate prescription medications, in the absence of positive hepatitis B surface antigen, positive HCV antibody, significant alcohol consumption, or hypotension. We assessed for the frequency of liver transplantation or death occurring within six months of the suspected DILI. 
Lammert and colleagues (3)

\section{Results}

Four thousand eight hundred and thirty seven individuals in suspected NAFLD cohort, 61,355 individuals in control group 1, and 47,869 individuals in control group 2 received a prescription for one of ten candidate medications. Their selected characteristics are shown in Table 1. The mean and median (range) baseline ALT levels in the suspected NAFLD cohort were $87 \mathrm{U} / \mathrm{L}$ and 73 (46-193) IU/L for the first value and $69 \mathrm{U} / \mathrm{L}$ and 58 (46-190) U/L for the second value. The frequency of prescriptions for various medications was generally consistent across these study groups, with cefazolin being the most and isoniazid being the least commonly prescribed medications. The frequency of suspected DILI in 4,837 individuals in suspected NAFLD cohort was $0.8 \%$ (40 out of 4,837 ) and it was significantly higher than in control group 1 (126 out of 61,355 [0.2\%], OR: 4.0, 95\% Cl: 2.8-5.8, $\mathrm{p}<0.001$ ) and control group 2 (96 out 47,869 [0.2\%], (OR: 4.17, 95\% Cl: 2.9-6.0), p<0.001) (Table 1). The mean (s.d.) ALT, Alk $P$, and total bilirubin levels of suspected DILI cases were $191 \pm 127 \mathrm{U} / \mathrm{L}, 197 \pm 144 \mathrm{U} / \mathrm{L}$, and $2.25 \pm 3.4 \mathrm{mg} / \mathrm{dL}$ in the suspected NAFLD group, $107 \pm 147.5 \mathrm{U} / \mathrm{L}, 251 \pm 170 \mathrm{U} / \mathrm{L}$ and $1.98 \pm$ $2.9 \mathrm{mg} / \mathrm{dL}$ in control group 1 , and $102 \pm 156 \mathrm{U} / \mathrm{L}, 251 \pm 179 \mathrm{U} / \mathrm{L}$, and $1.76 \pm 1.42 \mathrm{mg} / \mathrm{dL}$ in control group, respectively. The frequency of death or liver transplant within 6 months of suspected DILI was $25 \%$ in the CLD cohort (10 out of 40 ) and it was numerically but not statistically higher than in either control group 1 (21 out of 126 [16.6\%], $p=0.2$ ) or control group 2 (13 out of $96[13.5 \%], p=0.1$ ). (Table 1)

\section{Discussion}

This pharmaco-epidemiological study suggests that individuals with underlying chronic liver disease suggestive of NAFLD may be at higher risk for developing suspected DILI from prescription medications which are commonly implicated for causing DILI in the United States.

An important limitation of our study is that we defined the presence of chronic liver disease and suspected DILI utilizing surrogate criteria. However, we established two carefully 
Lammert and colleagues (4)

constructed algorithms to define CLD and suspected DILI in this study. Although we recognize that DILI can sometimes occur well beyond 3 months after initiating a culprit drug, we chose a 12 week latency period to strengthen the temporal relationship between candidate medication exposure and the onset of the suspected DILI events. Since we excluded individuals with hepatitis $\mathrm{C}$, hepatitis $\mathrm{B}$, or heavy alcohol consumption, our observations cannot be extended to individuals with CLD due to those etiologies. Our rationale behind excluding these etiologies was our intent to develop a CLD group that may mimic NAFLD. Interestingly, the signal we observed is consistent with an earlier study which suggested that patients with NAFLD had four fold higher risk for DILI compared to chronic hepatitis C.(1) In summary, these data suggest that individuals with suspected NAFLD may be at higher risk for suspected DILI. More studies are needed to confirm our observations. 
Lammert and colleagues (5)

\section{References}

1. Chalasani N, Bonkovsky HL, Fontana R, Lee W, Stolz A, Talwalkar J, Reddy KR, et al. Features and Outcomes of 899 Patients With Drug-Induced Liver Injury: The DILIN Prospective Study. Gastroenterology 2015;148:1340-1352.e1347.

2. Aithal GP, Watkins PB, Andrade RJ, Larrey D, Molokhia M, Takikawa H, Hunt CM, Wilke RA, et al. Case definition and phenotype standardization in drug-induced liver injury. Clin Pharmacol Ther 2011; 89: 806-815

3. Tarantino G, Conca P, Basile V, Gentile A, Capone D, Polichetti G, Leo E. A prospective study of acute drug-induced liver injury in patients suffering from non-alcoholic fatty liver disease. Hepatol Res 2007;37:410-415. 
Lammert and colleagues (6)

Table 1: Selected Clinical Characteristics of Individuals who received one of 10 candidate prescription medications $\mathbb{\|}$ in 3 Study Cohorts

\begin{tabular}{|c|c|c|c|}
\hline & \multicolumn{3}{|c|}{ Exposed to one of 10 candidates medications } \\
\hline & $\begin{array}{c}\text { Control } \\
\text { group } 1 \\
(n=61,355)\end{array}$ & $\begin{array}{l}\text { Suspected } \\
\text { NAFLD Cohort } \\
\qquad(n=4,837)\end{array}$ & $\begin{array}{l}\text { Control } \\
\text { group } 2 \\
(n=47,869)\end{array}$ \\
\hline Age (yr, mean \pm SD) & $55.8 \pm 18$ & $50.6 \pm 14.7$ & $56.7 \pm 18$ \\
\hline Females (\%) & 65 & 48 & 60 \\
\hline Ethnicity (Black/White) (\%) & $11 / 87$ & $8.6 / 90$ & $12 / 87$ \\
\hline $\mathrm{BMI}(\mathrm{kg} / \mathrm{m} 2$, mean $\pm \mathrm{SD})$ & $31 \pm 8.6$ & $32.1 \pm 8.7$ & $30.4 \pm 8.4$ \\
\hline AST at baseline (IU/L, mean \pm SD) & $21.5 \pm 11.5$ & $56 \pm 38$ & $20 \pm 11$ \\
\hline ALT at baseline (IU/L, mean \pm SD) & $19 \pm 8.2$ & $69 \pm 27$ & $17 \pm 5$ \\
\hline Alk $P$ at baseline (IU/L, mean \pm SD) & $74.5 \pm 29$ & $93 \pm 41$ & $73 \pm 27$ \\
\hline $\begin{array}{l}\text { Total bilirubin at baseline }(\mathrm{mg} / \mathrm{dL} \\
\text { mean } \pm \mathrm{SD})\end{array}$ & $0.6 \pm 0.5$ & $0.7 \pm 0.4$ & $0.6 \pm 0.34$ \\
\hline Presence of type 2 diabetes $^{\delta}(\%)$ & 24 & 34 & 24 \\
\hline Presence of hypertension ${ }^{\delta}(\%)$ & 50 & 56 & 51 \\
\hline Suspected DILI (\%) & 0.2 & 0.8 & 0.2 \\
\hline & & 0.001 & $P<0.001$ \\
\hline $\begin{array}{l}\text { Death or transplant }{ }^{\delta} \text { within } 6 \\
\text { months after suspected DILI } \\
\text { Onset (\%) }\end{array}$ & 16.6 & 25 & 13.5 \\
\hline & & & $P=0.1$ \\
\hline
\end{tabular}

Abbreviations: BMl: Body mass index; AST: Aspartate aminotransferase; ALT: Alanine aminotransferase; Alk P: Alkaline phosphatase.

I Ten candidate medications: Amoxicillin-clavulanate, isoniazid, nitrofurantoin, minocycline, trimethoprimsulfamethoxazole, ciprofloxacin levofloxacin, azithromycin, cefazolin, and diclofenac. 
Lammert and colleagues (7)

סHypertension was identified based ICD10 like 'I1\%' or ICD9 like '402\%', '403.\%' , '404.\%'; DM was identified based on ICD10 like 'E08\%, 'E09\%' , 'E10\%' , 'E11\%' or ICD9 like 'E250\%'; Transplantation was identified using either ICD9 V42.7 or ICD10 Z94.4, or following procedures $471.35,50.51,50.59$. 